Original Research Paper

\title{
Development of Electronic Control Circuits for WSN: Towards a Livestock Tracking and Identification System
}

\author{
Obakeng Maphane, Oduetse Matsebe and Molaletsa Namoshe \\ Department of Mechanical, Energy and Industrial Engineering, \\ Faculty of Engineering and Technology, \\ Botswana International University of Science and Technology, \\ Private Bag BO 016, Palapye, Botswana
}

Article history

Received: 6-07-2017

Revised:23-08-2017

Accepted: 11-09-2017

Corresponding Author:

Oduetse Matsebe

Department of Mechanical,

Energy and Industrial

Engineering,

Faculty of Engineering and

Technology, Botswana

International University of

Science and Technology,

Private Bag BO 016, Palapye,

Botswana

Email: matsebeo@biust.ac.bw

\begin{abstract}
Botswana's beef sector contributes $0.3 \%$ of the total world beef exports, i.e. US\$ 42.4 billion as of the year 2013 and her major market is the European Union (EU). Unfortunately, her failure to comply with the frequently changing European Commission (EC) export regulations on livestock tracking and identification means that beef export to this market is sometimes suspended. Research trends indicate that smart agriculture solutions have gained popularity in agricultural production and can help keep up with the frequently changing EC export regulations especially on active tracking and identification of livestock. It is against this background that this paper presents the development of electronic control circuits for Wireless Sensor Network (WSN) and Group Special Messaging (GSM) to be utilized in a livestock tracking and identification system. It is envisaged that the proposed solution would help fulfill potential changes of EC regulations. Proteus 8 software is used to develop the circuit models. The controller used for this work is Arduino microcontroller. Simulation results show that the XBee shields are able to communicate successfully. This communication forms the basis for the WSN. The results indicate that the developed electronic control circuits for the WSN are viable. Future work will focus on the development of a prototype that will be tested and validated in real environment.
\end{abstract}

Keywords: Wireless Sensor Networks, Internet of Things, Foot and Mouth Disease, Internet of Things, Livestock Tracking

\section{Introduction}

A study conducted by the International Trade Centre (ITC) on the review of the beef sector value chain, strategy and intervention for the Private Sector Development Program (PSDP) indicates that Botswana beef sector contributes $0.3 \%$ of the total world beef exports, i.e. US\$ 42.4 billion as of the year 2013. Her failure to comply with the frequently changing European Commission (EC) export regulations on livestock tracking and identification has sometimes led to the loss of major markets in the EU (Boy, 2013.). The study by ITC recommended the adoption of electronic ear tag system, Spongi form Encephalopathy (BSE) or Mad Cow disease and encouraged the use of technology to improve the out break in 1997 (Jin et al., 2006). The EU market issued regulation beef sector.
Traditionally, Batswana have and still use ear cuttings and branding to identify their livestock (Ndubo et al., 2012). They rely on word of mouth for tracking, ear cuttings descriptions, thigh brandings and livestock coat colour for identification purposes in Fig. 1 and Fig. 2.

These methods provide visible identification of livestock, they are cheap and easy to administer. Unfortunately, the methods do not provide tracking capability and history. They are easily tempered with and result in ambiguous ownership since they normally represent family identification. These methods were acceptable by the EC export regulations until the Bovine Spongiform Encephalopathy (BSE) or Mad Cow disease outbreak in 1997 (Jin et al., 2006). The EU market issued regulation EC820/97 which required member states to have a computerized identification and tracking system for beef going to the EU market (Ndubo et al., 
2012). As a result the Botswana Meat Commission (BMC) temporarily closed exports to EU while the Ministry of Agriculture (MoA) devised a system that would comply with the new regulations. The Department of Veterinary Services (DVS) under MoA responded to regulation EC820/97 with the introduction of Livestock Identification and Traceback System (LITS) program using reticular bolus Fig. 3 encasing a passive RFID chip for identification and trace back. The embedded RFID chip stores owner's details, animal's coat colour, sex, age, owner's brand number and location of the closest extension office to the cattle post (Barner Rasmussen et al., 2013). Some of the advantages of reticular bolus include; reduction in stock theft, unique animal identification, ability to trace back animal movements. It also stores the owner's and animal's details in an electronic database for historic purposes. The bolus system proved to be a challenge for the DVS, which is responsible for administering and educating farmers on the LITS program. The program was faced with challenges such as high administration costs for insertion and monitoring of boluses, lack of enough trained personnel to administer the boluses and it is a complex process farmers could not be taught in a short time, repeat insertions were also common because of lack of visibility, loss of tags by livestock, shortage of bolus tags limited the speed of program roll out to farmers hence failure to fulfil the EC export regulations.

In 2000, the EC released an updated regulation EC $1760 / 00$ which forced an upgrade to the program to meet the new regulations. Some of the requirements of the new regulations were:

- Bovine animals must be registered and individually identified with ear tags on each ear

- Individual passport of transport are required to accompany any bovine movements

- Computerized cattle tracing database in an attempt to meet these requirements

The DVS upgraded the LITS program from ingestible bolus to electronic RFID ear tags (Ndubo et al., 2012). The electronic ear tags in Fig. 4 and Fig. 5 are visible and easy to administer. The DVS managed to educate farmers on installation of these ear tags and eliminated dependency on DVS officials hence significantly reducing administration costs. The ministry was only responsible for stocking the ear tags and logging the numbers of sold tags in a centralized database. However, sold ear-tags do not necessarily mean they have been used to tag livestock and the DVS still relied on farmer to record the details and report them back for updating. This resulted in errors as some farmers tend to loose the records.

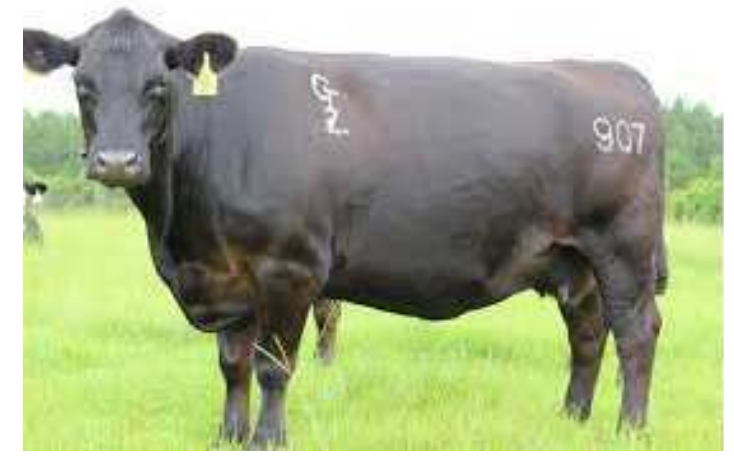

Fig. 1: Hot iron branding (CattleToday.com, 2013)

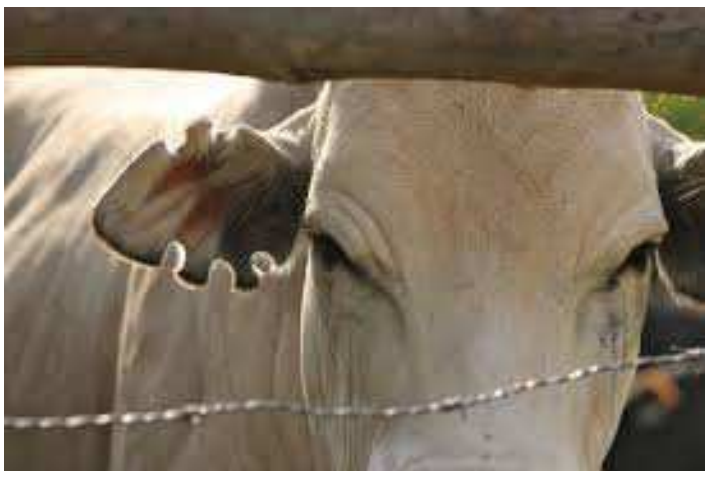

Fig. 2: Ear Cuttings (National Geographic, 2015)

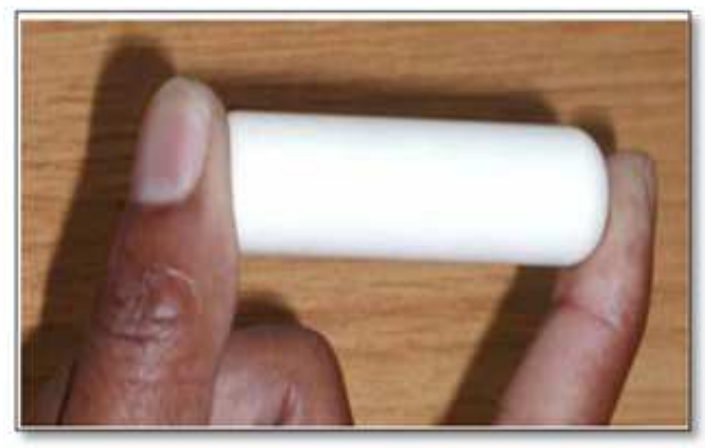

Fig. 3: Ingestible reticular bolus "Omang wa Dikgomo" (Department of Veterenary Services, 2005)

Some of the advantages of the RFID ear tags include; visible and unique identification, ability to trace back livestock, stores owner's details, information is stored in a centralized database for historic purposes, easy to remove tags from dead animals, it is user friendly and the tags are reasonably cheap. However, the RFID ear tags do not safe guard against stock theft, tags can be easily cut off, it does not provide constant monitoring of animal's movements; assumes livestock is within range of its zone, it can get lost if the animal gets caught in a fight or by tree brunches. The system only provides a trace back of livestock with gaps between readings or scannings, i.e. during vaccination periods, when they are transported across Foot and Mouth Disease (FMD) zones and during transportation to the BMC for sale. 


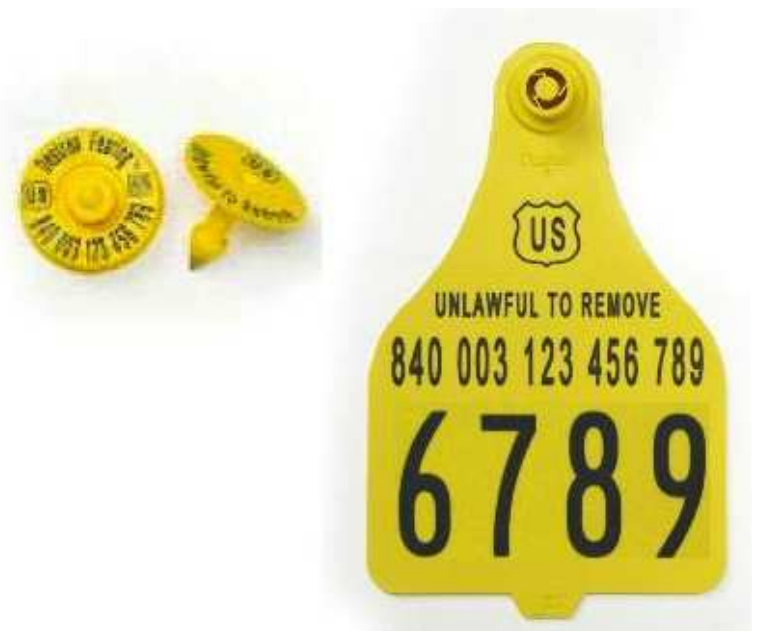

Fig. 4: Current electronic RFID ear tags (Omondi, 2015)

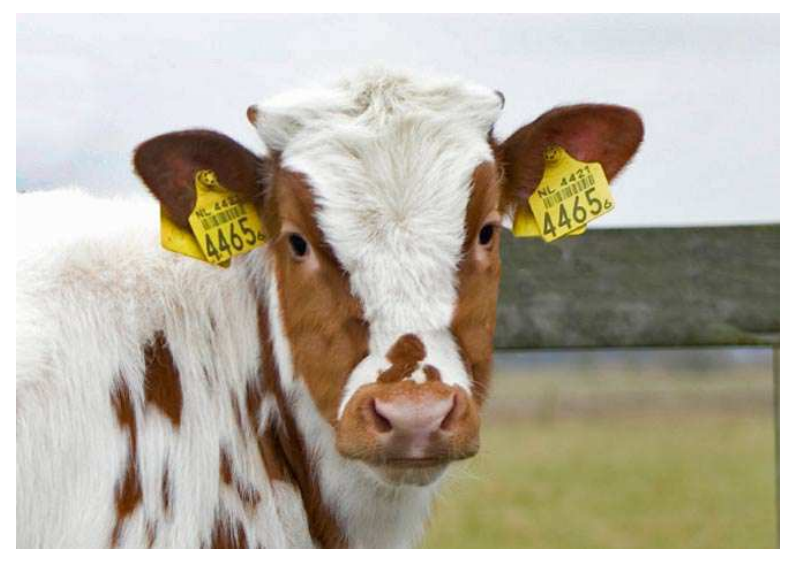

Fig. 5: Passive electronic RFID tag on a cow (Omondi, 2015)

Therefore, this system led to an inaccurate history of livestock movement over their lifespan. It is against this background that this research work proposes a solution that incorporates the current LITS features, and also enables real time tracking and identification of livestock using WSN, and the existing GSM network. The system uses a hybrid power module (solar and battery) for prolonged operation. However, the system will require access to the GSM network and an improved functionality that will help keep up with the frequently changing EC export regulations. The rest of the paper is structured as follows; section 2 reviews related works, section 3 outlines the methods and techniques, section 4 presents the results and discussions, sections 5 and 6 respectively presents the conclusion and future work.

\section{Literature Review}

A localization and monitoring system based on ZigBee protocol for cattle is presented in (Choi et al., 2013). In this work, Link Quality Indicator (LQI) accompanied with a modified Ratio metric vector
Iterations (RVI) routing algorithm for ranging is used. The authors use estimation and localization algorithms instead of a Global Positioning Satellite (GPS) which is a more precise localization method but expensive for livestock applications. A link of static anchors and mobile sensors attached to cattle ear tags encased in plastic casing and powered by cylindrical lithium ion battery are used. The anchor nodes are solar powered. Using Jennic JN5139 modules at 2.4Ghz, the research work manages to get distances of about $200 \mathrm{~m}$ in the lab, about $220 \mathrm{~m}$ in the field. The nodes can be detected from a height of $20 \mathrm{~cm}$ to $2 \mathrm{~m}$ above ground. Localization is achieved with an error of about $20 \mathrm{~m}$ using low cost hardware overhead. A system to remotely monitor the health of cattle using sensor network and Bluetooth as gateway or base station for telemetry is described in (Choi, 2013). The research work was motivated by restrictions and lack of an early warning system for mad cow disease in Canada. The system uses variable sensors to capture vital signs and to identify a threat to an outbreak. GPS is used for localization and trace back. The mobile sensors attached on the cow's ears contains a probe for temperature, pulse-oximeter, electronic belt, and respiration transducer for oxygen level, reticular bolus for core body temperature and Bluetooth transceiver for data transfer when the animal is in range of watering or feeding point. The data is then transferred to a workstation for computation and diagnosis. Unfortunately, the system could not be tested in the field but future modification included a short range telemetry link.

A system to monitor vital signs; temperature, heart rate, respiration and a tracking unit for cattle is presented in (Kwong et al., 2012). The system employs solar powered ear tags attached to cattle and rechargeable batteries for prolonged life span of the WSN. A combination of static nodes and mobile nodes using triangulation through RSSI localization and estimation are used. A low power WSN with a graphical user interface is developed in a MATLAB environment. This provides positioning information through triangulation within a fenced area. A ZigBee-based protocol is used. The authors propose the use of RFIDs and other sensors to improve the applicability of the system depending on farmer's requests. The conclusion made here is that a wireless system is viable for cattle monitoring and state of health research as well as natural patterns or cattle behaviour.

Barner Rasmussen et al. (2013), researchers adapted a WSN application for dairy farming in an enclosed area. The system developed is low cost, has a low power consumption sensor node and enables real time communication using channel bandwidth and radio frequency. Belts wrapped around the targeted animal's neck with two antennas on sides are used, utilizing MICA and MICAz nodes powered by AA batteries. Various frequencies from $315 \mathrm{MHz}$ to $2.4 \mathrm{GHz}$ were considered, 
and a penetration depth of those frequencies. The main challenge reported is adapting wireless sensor networks to support nodes mobility caused by animal movements. A cluster based routing protocol and architecture for tracking livestock with focus on scalability, mobility and energy efficiency is developed in (Lin et al., 2004). The research work indicates that large herds of livestock and vast pastureland livestock herding are a major limitation in open ranges, the application of WSN is also limited by scalability and energy efficiency (Ficek et al., 2013); hence the authors use a clustering algorithm for the architecture while nodes are subdivided by the introduction of mobile sinks through GSM network. Existing cluster based routing protocols; LEACH-Mobile Enhanced and Mobile Sink based Routing Protocol (MSRP) based on the remoteness mobility metric are employed.

\section{Methodology}

Proteus 8 software is used to model and simulate the electronic control circuits for the WSN. The major advantage of using Proteus 8 is that it has the ability to render third party libraries such as those of Arduino Microcontrllers, GPS receiver, XBee shield and SIM900 a GSM shiled. The controller used for this work is the Arduino MCU since it is a programmable off-shelf component and it can be uploaded with Arduino emulator code that will run on the physical microcontroller without modifications.

\section{Mobile Node to Mobile Node Radio Transmission Model}

The model in Fig. 6 shows the radio connection between two XBee shields, these represent the mobile nodes in a mesh WSN. The XBee modules are connected to Arduino MCU, virtual monitors display the shared packets during transmission.

\section{GPS Receiver Connection Model}

Figure 7 shows a virtual GPS receiver connected to a virtual Arduino Uno MCU, and a virtual terminal monitor. This represents the reception of GPS coordinates which are displayed on the terminal monitor. This scenario arises when a static node is connected to the network.

\section{Mobile to Static Node Radio Connection}

Figure 8 depicts the mobile node to static node connection. The mobile node consists of the Arduino pro mini and XBee shield while the static node is made up of XBee shield, GPS receiver, Sim900D Shield, Arduino MCU and a monitor. This represents a connection between an ear-tag and static node. The packets being shared are displayed on the monitor.

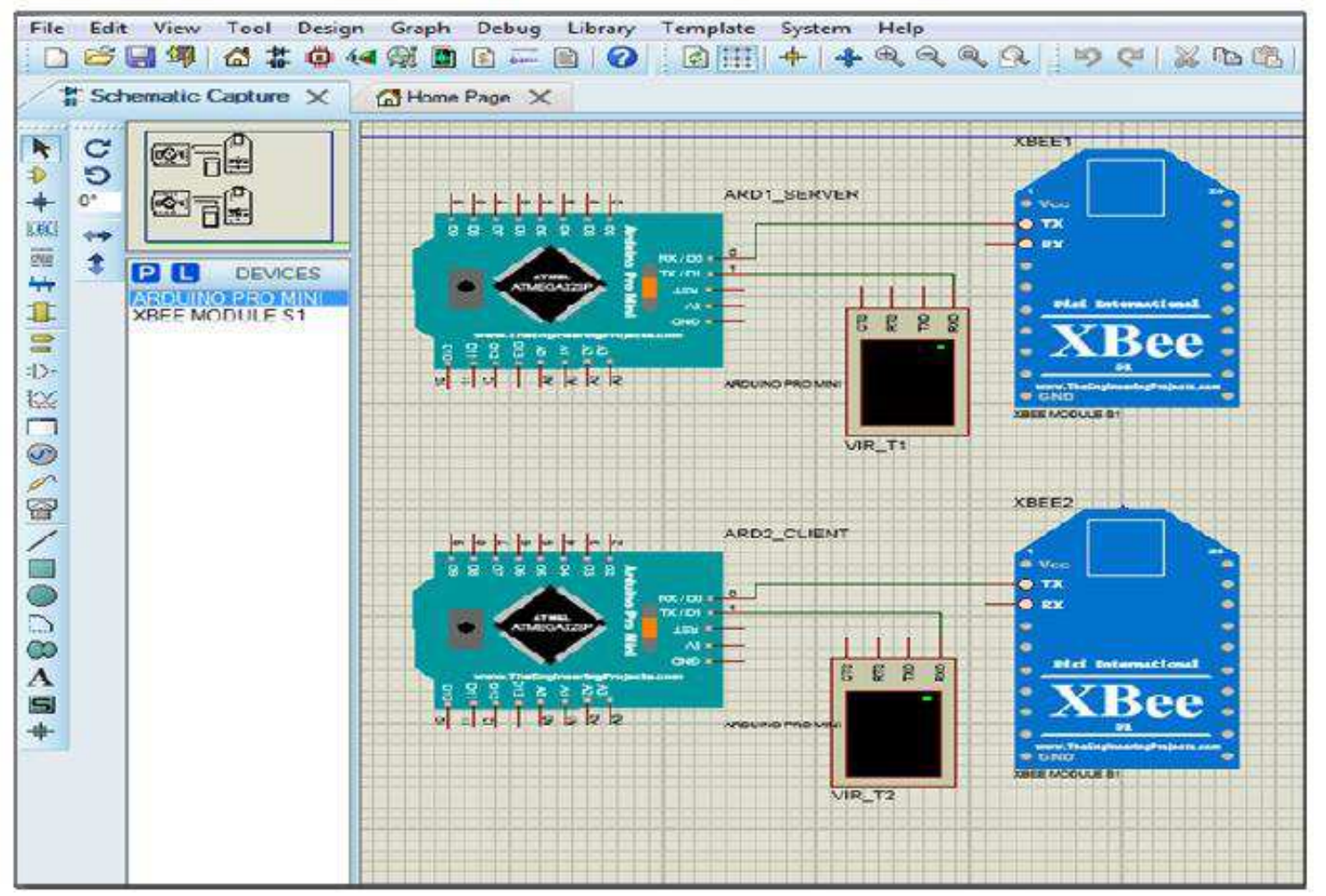

Fig. 6: Radio Connection between XBee Shields Nodes 


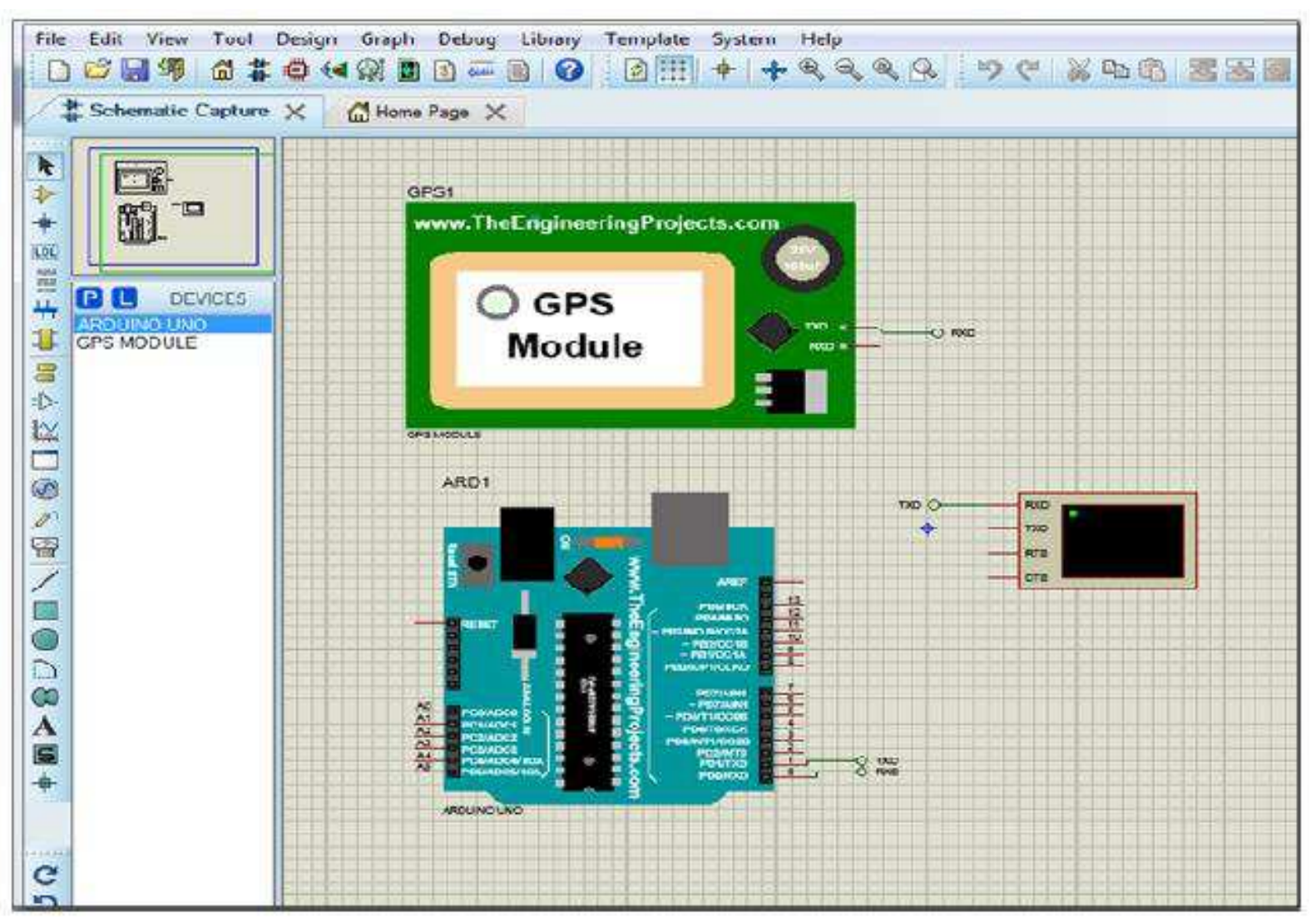

Fig. 7: Virtual GPS receiver Model

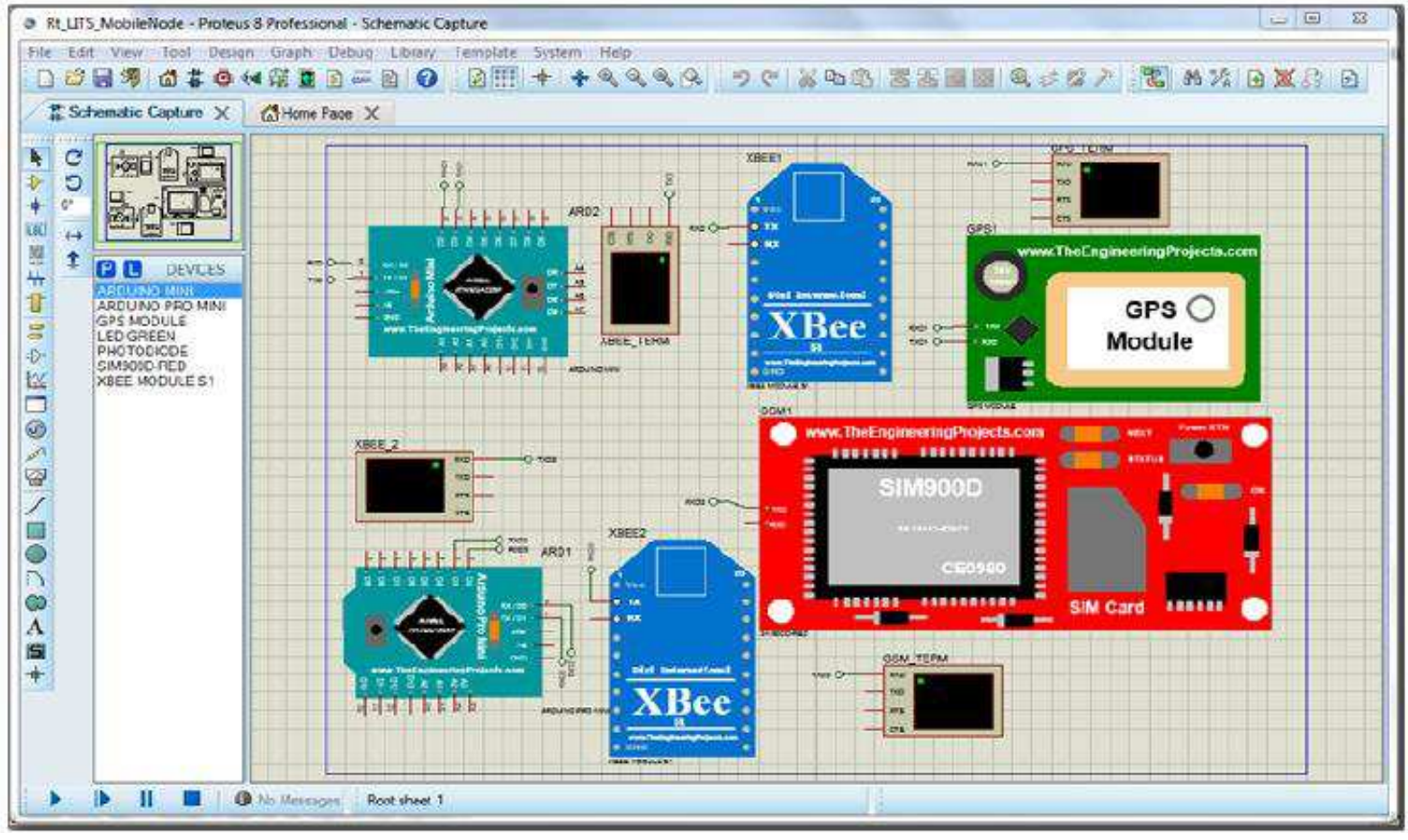

Fig. 8: Mobile node to static node model 


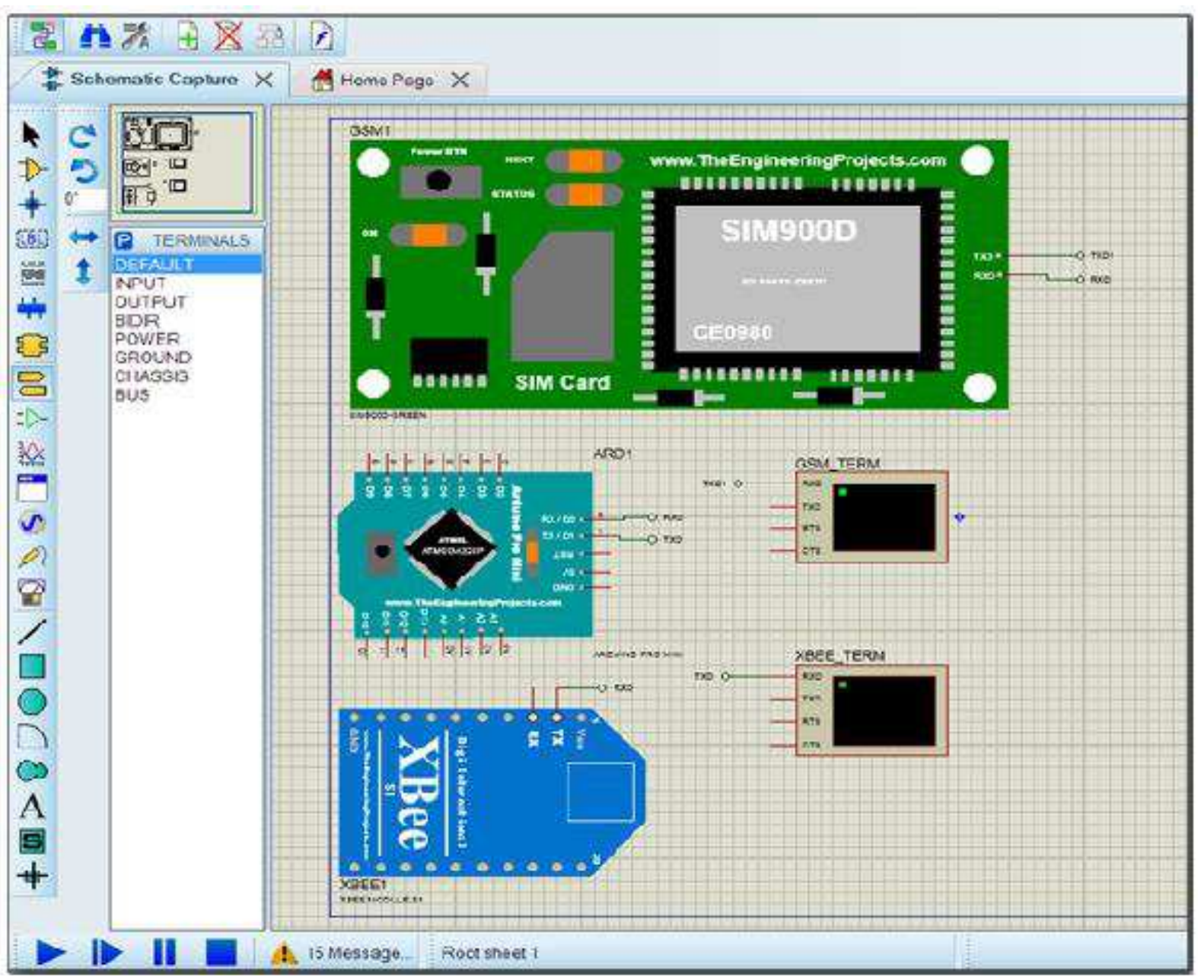

Fig. 9: Static Node to BST Connection Model

\section{Static Node to GSM Connection}

Figure 9 shows the connections between static node and mobile node. Packets received from the mobile node are sent to the base station or gateway for storage in the database. This represents the connection between WSN and GSM networks.

\section{Results}

This section presents the simulation results for the different circuit models.

\section{Mobile Node to Mobile Node}

Figure 10 shows successful communication between the XBee shields.

\section{GPS Receiver}

Figure 11 shows the GPS coordinates received.

\section{Static Node to Base Station Connection}

Data packets shared between the components is displayed in Fig. 12.

\section{Discussion}

The wireless shields (Fig. 10) create a Personal Area Network between them by exchanging data. This communication indicates a building block for the mesh WSN architecture. The GPS coordinates received (Fig. 11) are stored for further processing. These are fixed locations of the static nodes and are used to triangulate distance from the mobile nodes.

The static node (Fig. 12) computes distance information from radio signals obtained through the XBee shield. This information is then sent to the GSM terminal through the shared Arduino memory. The identifier variables; owner and livestock information shown in Fig. 13 are shared with XBEE TERM_2 connected to the static node. The MCU then computes the livestock $(x, y)$ positions from range data. The information is then appended to the data packet and passed to GSM_TERM. 


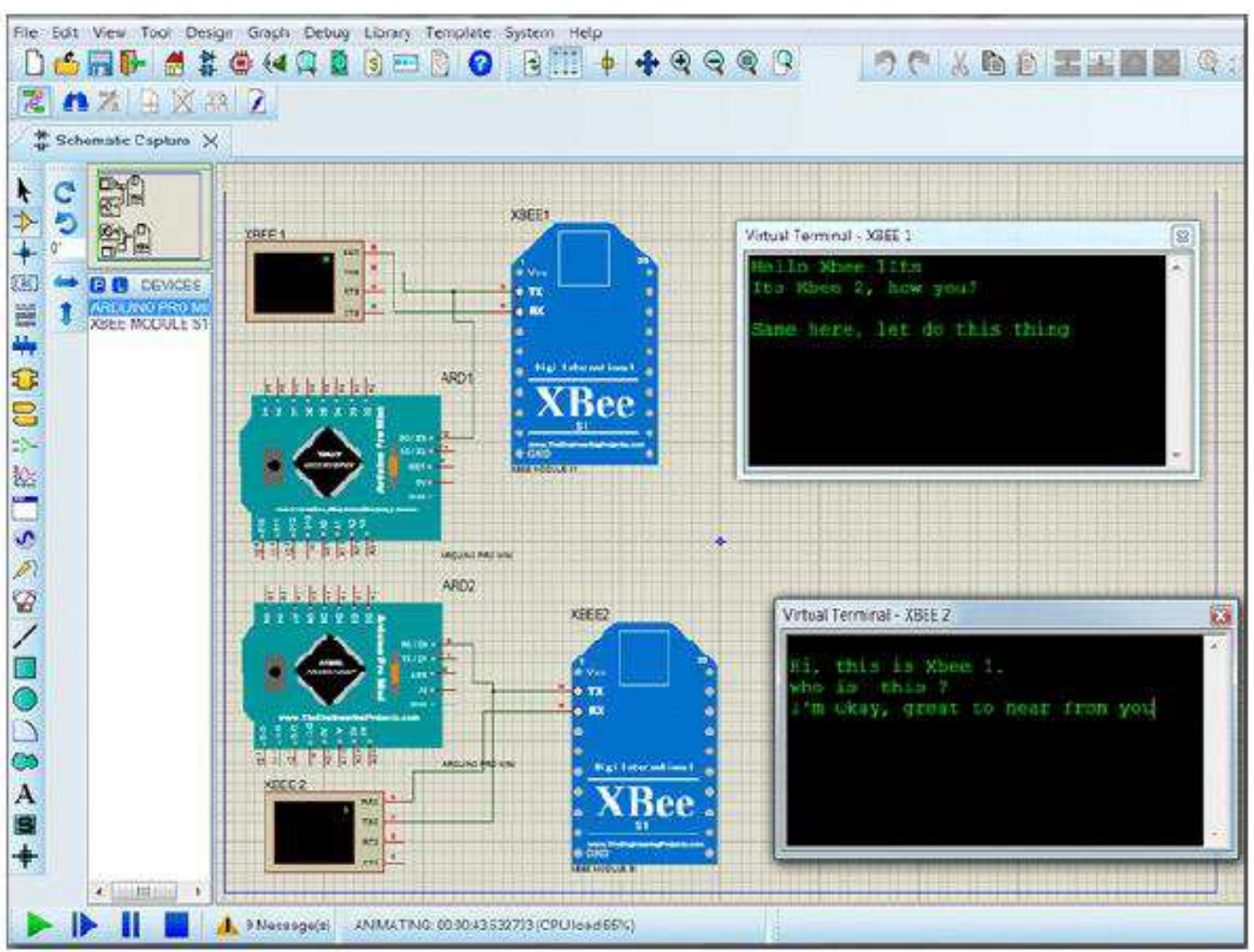

Fig. 10: Mobile Nodes Exchange through XBee Shields

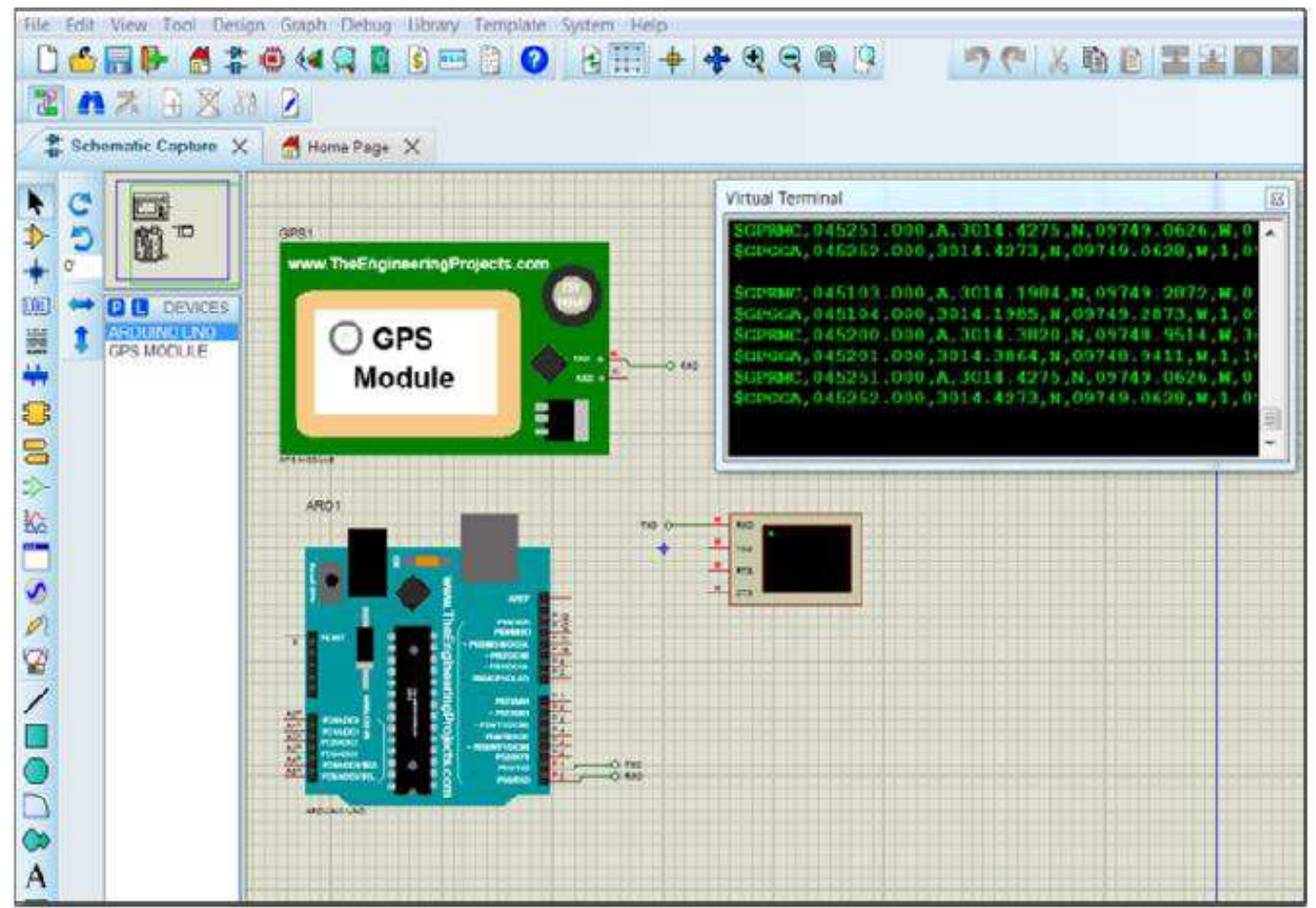

Fig. 11: Simulated GPS coordinates 


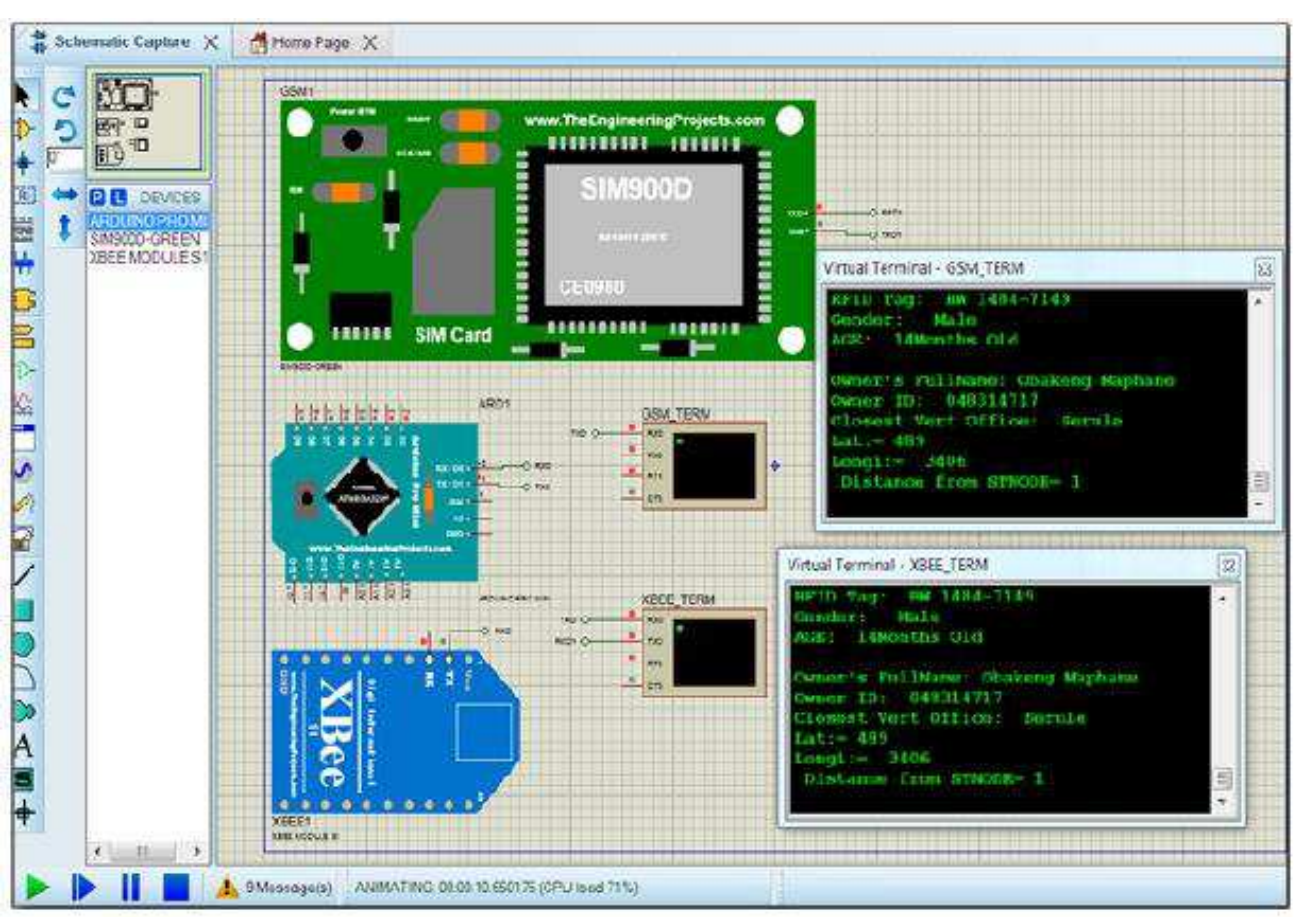

Fig. 12: Static sensor node to BST connection

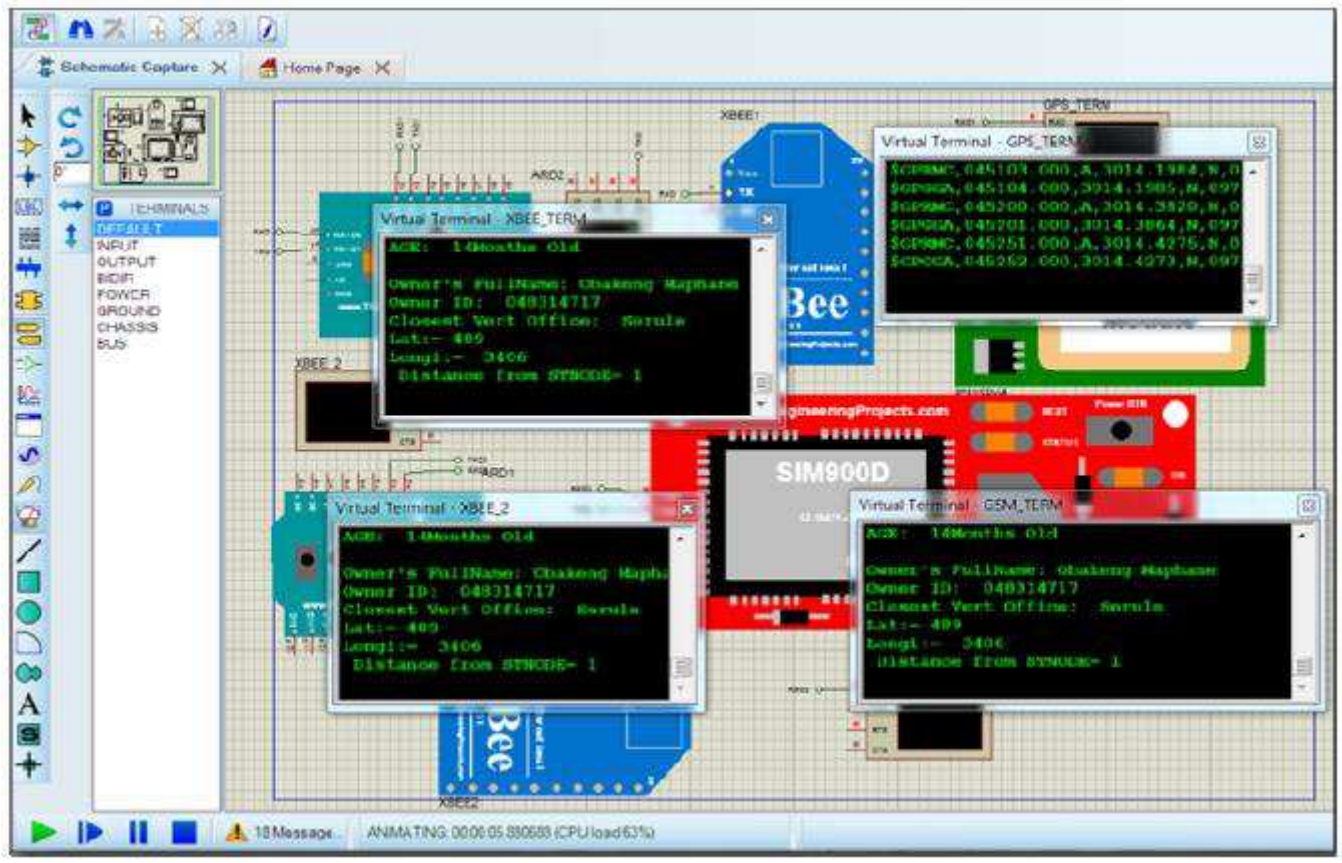

Fig. 13: Mobile to static connection

\section{Conclusion}

This paper presents the development of electronic control circuits for the WSN that will be utilised in a livestock tracking and identification system. Proteus 8 software is used to model and simulate the mobile node to mobile node radio transmission, GPS receiver connection, mobile to static node radio connection and static node to GSM connection. Simulation results show that the XBee shields are able to communicate successfully; this communication forms the basis for the WSN. GPS coordinates are received and stored for 
further processing. The static sensor nodes are able to compute distance information from the radio signals and the information is then passed to the GSM terminal. Identifier variables for owner and livestock are generated. Livestock(x,y) positions are also computed from range data received. These results indicate that the developed electronic control circuits for the WSN are viable. The complete system would improve livestock tracking and identification and hence keep up with the frequently changing EC export regulations.

\section{Further Study}

Future work will focus on the development of a prototype that will be tested and validated in real environment.

\section{Acknowledgement}

We express our appreciation to Dr. M.A Zungeru for his advice and to the Botswana International University of Science and Technology's for funding this research work.

\section{Author's Contributions}

Mr Obakeng Maphane: Participated in the conceptualisation of the research, collection of data, modelling and simulation, analysis and interpretaion of the results, drafting and reviewing of the manuscript. He approves this version of the document to be submitted.

Dr. Oduetse Matsebe: Is the supervisor and coordinator of the research. He participated in the conceptualisation, coordination and designing of the research, contributed to the the analysis and interpretation of results. He also drafted and reviewed the manuscript. He approves this version of the manuscript to be submitted.

Dr. Molaletsa Namoshe: Is the co-supervisor of the research. He participated in the conceptualisation and designing of the research, contributed to the the analysis and interpretation of results. He also reviewed the manuscript. He approves this version of the manuscript to be submitted.

\section{Ethics}

The are no ethical issues.

\section{References}

Barner Rasmussen, H., L.E. King, F.W. Ihwagi and E.A. Raizmana, 2013. Feasibility study on the spatial and temporal movement of Samburu Cattle and wildlife in Kenya Using GPS radio-tracking, remote sensing and GIS. Preventive Vet. Med., 111: 76-80.

DOI: $10.1016 /$ j.prevetmed.2013.04.007
Boy, R.L., 2013. Cattle traceability-a threat to sustainable suppy of beef to EU: A Botswana meat commssion. Eur. J. Logistics Purchasing Supply Chain Management, 1: 1-9.

CattleToday.com, 2013. Branding cattle.

Choi, S.E.N.J.Y., 2013. Cluster-based routing protocol in wireless sensor networks for tracking livestock in Mongolian normadic herding. Proceedings of the 8th International Forum on Strategic Technology, pp: 163-167.

Choi, S.G., E. Norinpel and J. Yoo, 2013. Cluster-based routing protocol in wireless sensor networks for tracking livestock movements in Mongolian nomadic herding. Proceedings of the 8th International Forum on Strategic Technology, 28 June-1 July 2013, IEEE Xplore Press, Ulaanbaatar, Mongolia pp: 163-167. DOI: 10.1109/IFOST.2013.6616879

Department of Veterenary Services, 2005. Omang wa Dikgomo. Gaborone.

Ficek, M., T. Pop and L. Kencl, 2013. Active tracking in mobile networks: An in-depth view. Comput. Netw., 57: 1936-1954. DOI: 10.1016/j.comnet.2013.03.013

Jin, K.S., J.C. McEachen and G. Singh, 2006. RF characteristics of Mica-Z wireless sensor network motes. Proceedings of the 49th IEEE International Midwest Symposium on Circuits and Systems, Aug. 6-9, IEEE Xplore press, Puerto Rico. DOI: 10.1109/MWSCAS.2006.382218

Kwong, K.H., TT. Wua. H.G. Goha, K. Saslogloua and B. Stephen et al., 2012. Practical considerations for wireless sensor networks in cattle monitoring applications. Comput. Electronics Agric., 81: 33-44. DOI: 10.1016/j.compag.2011.10.013

Lin, D.B., R.T. Juang and H.P. Lin, 2004. Mobile location estimation and tracking for GSM systems. Proceedings of the 15th IEEE International Symposium on Personal, Indoor and Mobile Radio Communications, Sept. 5-8, IEEE Xplore press, Spain, pp: 2835-2839. DOI: 10.1109/PIMRC.2004.1368838

National Geographic, 2015. Cow identification. National Geographic Society.

Ndubo, N.S., T. Ditshupo, J.B. Ntesang and J.C. Moreki, 2012. Cattle identification and traceability in Botswana. J. Anim. Sci. Advances, 2: 925-933.

Omondi, J., 2015. Electronic Micro chipped ear tags redefine livestock branding. 